\title{
ON THE STATIC SOLUTIONS IN GRAVITY WITH MASSIVE SCALAR FIELD IN THREE DIMENSIONS
}

\author{
G. de Berredo-Peixoto ${ }^{1}$ \\ Department of Physics, University of Alberta, Edmonton, Canada. \\ 412 Avadh Bhatia Physics Laboratory, Edmonton, AB, T6G 2J1 Canada.
}

\begin{abstract}
We investigate circularly symmetric static solutions in three-dimensional gravity with a minimally coupled massive scalar field. We integrate numerically the field equations assuming asymptotic flatness, where black holes do not exist and a naked singularity is present. We also give a brief review on the massless cases with cosmological constant.
\end{abstract}

Keywords: Three-dimensional gravity, black hole, scalar field, naked singularity.

PACS: 04.50.+h; 04.70.-s.

\section{Introduction}

Black holes (BH) are one of the most intriguing features of General Relativity. Although they are defined by geometrical concepts ${ }^{2}$, they possess remarkable thermodynamical properties, according to the results of Hawking [3]. These properties arise due to quantum effects of matter in a curved spacetime (Hawking radiation), providing strong identification between the laws of thermodynamics and the classical energy formula in General Relativity (see [4]), which had been regarded only as a mathematical relationship.

In principle, the $\mathrm{BH}$ thermodynamics is not described by some statistical formalism, as in the case of standard thermodynamics, but it is derived from geometrical degrees of freedom. For example, the $\mathrm{BH}$ temperature is usually given by the surface gravity (which is a geometrical object) for any reasonable matter content. However, it is not clear how the $\mathrm{BH}$ termodynamics can be affected if one considers a gravity theory with additional degrees of freedom, besides the metric. One can consider, for example, the Poincaré Gauge Theory of Gravity (PGTG) [5], which includes torsion in the physical description. For a review concerning theories with torsion, see [6].

There are many possible approaches to this investigation. One could consider theories with algebraic torsion (non-dynamical torsion). In this case, torsion is trivial in the absence of spin fields. Thus, given a $\mathrm{BH}$ vacuum solution, all the consequent thermodynamics is exactly the same as in General Relativity. On the other hand, one can consider more

\footnotetext{
${ }^{1}$ Present address: Departamento de Física, Universidade Federal de Juiz de Fora, Juiz de Fora, MG, Brazil 36036-330. Electronic address: guilherme@fisica.ufjf.br

${ }^{2}$ see $[1,2]$ for a pedagogical introduction to General Relativity and black holes.
} 
interesting models, with dynamical torsion. One can find spherically symmetric BH solutions with dynamical torsion in the four dimensional $R^{2}$-Gravity framework, in the teleparallel limit [7] as well as in the more general cases [8, 9]. In Ref. [9] one can find a stationary BH solution in the quadratic Poincaré Gravity.

In this paper we consider three dimensional gravity coupled to scalar field, with special attention on black holes configurations. The first black hole solution in three dimensions was discovered by Bañados, Teitelboim and Zanelli [10]. Among many interesting properties of the BTZ black holes (see the review [11]), one can mention that it is a string solution in the low energy regime [12]. In addition, the BTZ black hole is achieved as the final state of the spacetime with collapsing matter [13], where one can find equilibrium conditions for stable stars with matching conditions with the exterior BTZ solution [14]. See [15] for collapsing solution with scalar field without the BTZ solution as the final state. In the papers of Refs. $[16,17]$ the generalization to charged stationary solutions is investigated. One can find also the magnetic counterpart of the BTZ static solution[17, 18, 19], as well as its rotating solution [20]. The paper in Ref. [21] extends the rotating electrically charged BTZ solution to include Brans-Dicke theory. Solutions for minimally coupled massless scalar field with non-trivial cosmological constant can be found in Ref. [22], without black holes. There are two classes of solutions: one with naked singularity and other which is geodesically complete. In addition, one can find a one-parameter family of charged black holes for the 3D-dilaton gravity [23], as well as its uncharged rotating version [24], where the black hole is specified by the mass, angular momentum and the dilaton coupling parameters. A nontrivial black hole solution was found for a minimally coupling scalar field and a class of potentials [25]. Recently, Garcia et al have developed a three-dimensional Poincaré Gauge Theory of Gravity including the Chern-Simons term [26]. Maluf and Sousa [27] considered the restricted teleparallel version (with a Lagrangian quadratic in the torsion tensor) to describe a black hole solution following the Hamiltonian fomalism.

This paper is organized as follows. In Section 2, we introduce the general action for gravity minimally coupled to a massive scalar field, consider some special cases avaiable in the literature, and present the field equations for a particular action, with vanishing cosmological constant. We show also a possible interpretation for the action in the Appendix. In Section 3, we find a solution by numerical integration, which has a naked singularity. We show that BH solutions are absent (what is related to the no-hair theorems [28]). Finally, in Section 4, we give our conclusions.

\section{Action and field equations}

Let us introduce the general action with a scalar field (minimally coupled to gravity) which has the following form:

$$
S=\int d^{3} x \sqrt{-g}\left\{\frac{1}{\kappa} R-\nabla_{\mu} \phi \nabla^{\mu} \phi-M^{2} \phi^{2}-\frac{1}{4} e^{a \phi} F_{\mu \nu} F^{\mu \nu}+V[\phi]\right\},
$$

where $\kappa$ is a coupling constant ${ }^{3}$. This action describes a wide class of possible theories, and a complete study is still not avaiable. Many authors have investigated some sectors of the action (1). For instance, Chan and Mann [23] considered circularly symmetric static solutions

\footnotetext{
${ }^{3}$ Here, $\kappa$ has dimensions of mass ${ }^{(-1)}$, and, in principle, it is not related to Newton's constant, due to the lack of Newtonian limit in 3D-Einstein's theory [29].
} 
in the massless case, $M=0$, and they found a class of charged black hole configurations, with $V[\phi]=-2 e^{b \phi} \Lambda$. The rotating black holes were also discovered in the uncharged case [24]. If we let $b=0, M=0$ and $F_{\mu \nu}=0$, the resulting action is the one considered by Clément and Fabbri [22], and their solutions can not be achieved continuously from the dilaton solution [23] by the procedure $b \rightarrow 0$. We are going to investigate the uncharged version of (1), with $V[\phi]=0$ and $M \neq 0$ :

$$
S=\int d^{3} x \sqrt{-g}\left\{\frac{1}{\kappa} R-\nabla_{\mu} \phi \nabla^{\mu} \phi-M^{2} \phi^{2}\right\} .
$$

Note that the massive case are not convenient for searching a black hole solution by the virtue of the non-scalar hair theorem [30]. Surprisingly, no one has so far investigated the solutions for the theory (2), which is relatively simple in contrast to the more complicated cases already known in the literature. Let us remark that one should not expect to cover other known solutions by taking $M \rightarrow 0$ (e.g. Ref. [22] with $\Lambda=0$ ).

One should mention that the motivations for studying theories with scalar field varies from the Standard Model to Cosmology, as well as some aspects of the low energy regime of string theory. In the Appendix, we give a possible physical interpretation of the scalar field in the action (2).

\section{$2.1 \quad$ Field equations}

We are looking for circularly symmetric static solutions of theory (2), thus there are two Killing fields, $\partial / \partial t$ and $\partial / \partial \theta$ and the metric is given by

$$
d s^{2}=-f(r) d t^{2}+\frac{g(r)}{f(r)} d r^{2}+r^{2} d \theta^{2} .
$$

The Killing field $\partial / \partial t$ is hypersurface orthogonal, because the metric is static. Otherwise, the line element (3) would have crossing terms of the type $h(r) d t d \theta$.

Varying the action $(2)$ with respect to the field variables $\left(g_{\mu \nu}, \phi\right)$, one can find the field equations

$$
R_{\mu \nu}-\frac{1}{2} g_{\mu \nu} R=\kappa T_{\mu \nu}
$$

and

$$
\square \phi-M^{2} \phi=0
$$

where the momentum-energy tensor is given by

$$
T_{\mu \nu}=\nabla_{\mu} \phi \nabla_{\nu} \phi-\frac{1}{2}\left(g_{\mu \nu} \nabla_{\alpha} \phi \nabla^{\alpha} \phi+M^{2} g_{\mu \nu} \phi^{2}\right)-\frac{1}{\kappa} g_{\mu \nu} \Lambda
$$

and the operator $\square$ stands for $g^{\alpha \beta} \nabla_{\alpha} \nabla_{\beta}$. Here we introduced, for the sake of generality, the cosmological constant through the procedure $e^{b \phi} \rightarrow 1 / \kappa$ in (1).

After some calculations, one can achieve the following independent field equations (assuming $\phi=\phi(r))$

$$
\frac{f^{\prime}}{2 f r}-\frac{g^{\prime}}{2 g r}=-\frac{1}{2} \kappa\left(\phi^{\prime}\right)^{2}-\frac{1}{2} \kappa \frac{g}{f} M^{2} \phi^{2}-\frac{g}{f} \Lambda ;
$$




$$
\begin{aligned}
& -\frac{f^{\prime}}{2 f r}=-\frac{1}{2} \kappa\left(\phi^{\prime}\right)^{2}+\frac{1}{2} \kappa \frac{g}{f} M^{2} \phi^{2}+\frac{g}{f} \Lambda ; \\
& \phi^{\prime \prime}+\left(\frac{f^{\prime}}{f}-\frac{g^{\prime}}{2 g}+\frac{1}{r}\right) \phi^{\prime}+\frac{g}{f} M^{2} \phi=0,
\end{aligned}
$$

where $f^{\prime}$ means $d f / d r$. After some manipulations, we obtain the equivalent set of field equations

$$
\begin{gathered}
\frac{g^{\prime}}{2 g r}=\kappa\left(\phi^{\prime}\right)^{2} ; \\
-\frac{f^{\prime}}{2 f r}=-\frac{1}{2} \kappa\left(\phi^{\prime}\right)^{2}+\frac{1}{2} \kappa \frac{g}{f} M^{2} \phi^{2}+\frac{g}{f} \Lambda ; \\
\phi^{\prime \prime}-\kappa \frac{g}{f} M^{2} \phi^{2} \phi^{\prime} r-2 \frac{g}{f} \Lambda \phi^{\prime} r+\frac{1}{r} \phi^{\prime}+\frac{g}{f} M^{2} \phi=0 .
\end{gathered}
$$

Notice that by (10) and (12), solutions of the type $g(r)=$ const. are possible only if the field $\phi(r)$ is trivial, producing thus the well known static BTZ black hole solution [10] (See [11] for a review on the classical and quantum properties of the BTZ black hole).

\section{Numerical integration}

Before considering general solutions, let us investigate some particular cases to achieve more insight in the search for the general solution. In the case $M=\Lambda=0$, equation (12) reads

$$
\phi^{\prime \prime}+\frac{1}{r} \phi^{\prime}=0
$$

which has the solution

$$
\phi(r)=Q \ln \frac{r}{r_{0}}
$$

where $Q$ and $r_{0}$ are integration constants, with canonical dimensions $+1 / 2$ and -1 respectively. Other equations can be integrated, resulting in the following solution:

$$
\begin{gathered}
g(r)=\gamma r^{2 \alpha}, \text { with } \alpha:=\kappa Q^{2} \text { and } \\
f(r)=\delta r^{\alpha},
\end{gathered}
$$

where $\gamma$ and $\delta$ are integration constants with canonical dimensions $2 \alpha$ and $\alpha$. In this solution, the diverging scalar field provides diverging metric functions $f(r)$ and $g(r)$, far away from the origin, $r>>1$, with no horizon formation. In the flat background, $\phi$ has the same solution (14). Now consider the Minkowski spacetime with $M \neq 0$. In four dimensions, the 
solution is the well known meson field, $\phi=Q \exp (-M r)$. In our case, the equation for $\phi$ is a Bessel equation, with the modified Bessel function of the second kind as a suitable solution,

$$
\phi(r)=Q K_{0}(M r)
$$

with similar asymptotic behaviour to the meson solution in four dimensions.

We cannot find analytical solutions for the general case with $\Lambda=0$. Thus we integrate numerically using the software $\mathrm{MAPLE}^{4}$ (See, for instance, the book [32]). We first guess suitable initial values for the fields at infinity and then integrate from that point to the origin. As the mass is non-trivial, one can suppose that the scalar field decays with $r$. At infinity, we assume that the metric is approximately free from the influences of the scalar field, thus the metric at infinity can be regarded as the vacuum solution of the Einstein's equations. The appropriate ansatz for the metric at infinity is (see [29])

$$
d s^{2}=-d t^{2}+b d r^{2}+r^{2} d \theta^{2}
$$

which describes a flat spacetime with conical singularity at the origin $(b=$ constant). To make this supposition, we assume that the solution is asymptotically flat. The field equation for $\phi(r)$ in this background has solution $\phi(r)=Q K_{0}(M \sqrt{b} r)$. Let us remark that this ansatz is valid only in the asymptotic region; thus, despite this function is singular at the origin, this feature may not be true for the actual solution satisfying $\phi(r) \sim K_{0}(M \sqrt{b} r)$ far from the origin. We choose $r=30$ as the infinity and set $Q=2, M=0.1, b=1.1, f(30)=1$, $g(30)=b, \phi(30)=Q K_{0}(30 M \sqrt{b})$ and $\phi^{\prime}(30)=d \phi /\left.d r\right|_{r=30}$. The numerical integration carried out is described by the plotting on Figure 1 . As we increase the parameter $M$, the deviations of functions $f(r)$ and $g(r)$ from the vacuum solution, near origin, are decreased even more. These deviations are continuously increased if the scalar "charge", $Q$, is increased (as far as the value $r=30$ can be regarded as infinity with a negligible error). There is no black hole, as shown by Figure 1.

One can also integrate from the origin, starting from a suitable initial conditions. To find them, one has to guess an approximate solution near $r=0$. If we assume regularity in the origin, we suppose a power series in $r$ for the variables:

$$
\begin{gathered}
f \approx f_{0}+f_{1} r+f_{2} r^{2}+\ldots ; \quad g \approx g_{0}+g_{1} r+g_{2} r^{2}+\ldots ; \\
\phi \approx \phi_{0}+\phi_{1} r+\phi_{2} r^{2}+\ldots
\end{gathered}
$$

Notice that this guess has a limited importance, specially if some black hole solution is supposed to be found. However, it is a possible procedure of numeric integration from the origin.

One can verify that these solutions satisfy the field equations up to $O\left(r^{2}\right)$, if some constraints between the constant parameters are taken into account. After that, we find the following approximate solution for small values of $r$ :

$$
f(r)=a-\frac{1}{2} b q^{2} r^{2} ; \quad g(r)=b ; \quad \phi(r)=q-\frac{1}{4} \frac{b q}{a} r^{2}
$$

\footnotetext{
${ }^{4}$ The reader can obtain useful information about the numerical integration applied to General Relativity in several papers, say, for example, in papers of Ref. [31].
} 
where $a, b$ and $q$ are constant parameters. Thus, there is a three-parameter family of possible solutions near the origin. As the asymptotic solution at infinity has two parameters, only a sub-family of the tree-parameter family would produce (in principle) a convergent solution which coincides with the solution at infinity. However, we shall show that this three-parameter family is incompatible with the asymptotic flatness, so both families are actually distinct. Now, let us consider an approximate solution near an hypothetic horizon. One can write the following ansatz (assuming regularity of all functions at the horizon, $r_{h}$ )

$$
\begin{aligned}
& f \approx f_{0}\left(r-r_{h}\right)+f_{1}\left(r-r_{h}\right)^{2}+\ldots ; \\
& g \approx g_{0}+g_{1}\left(r-r_{h}\right)+g_{2}\left(r-r_{h}\right)^{2}+\ldots ; \\
& \phi \approx \phi_{0}+\phi_{1}\left(r-r_{h}\right)+\phi_{2}\left(r-r_{h}\right)^{2}+\ldots
\end{aligned}
$$

Again, one substitute these expressions into the field equations and demand that they must be satisfied up to the second order in $\left(r-r_{h}\right)$. We can achieve the following solution:

$$
f=a\left(r-r_{h}\right)^{2} ; \quad g=b+c\left(r-r_{h}\right)^{2} ; \quad \phi=\phi^{\prime}=0 .
$$

Actually this solution corresponds to the trivial scalar field case, and should not be considered. Now we shall show that no black holes are possible if the spacetime is asymptotically flat $^{5}$, unless the scalar field is trivial.

\subsection{No massive scalar hair}

Consider the static circularly symmetric line element, in some appropriate coordinate system,

$$
d s^{2}=-U^{2}(x) d t^{2}+d x^{2}+R^{2}(x) d \theta^{2} .
$$

The minimally coupled scalar field, $\phi(x)$, is a solution of (5):

$$
\frac{1}{U R}\left(U R \phi^{\prime}\right)^{\prime}=M^{2} \phi
$$

where the prime means derivative with respect to $x$. Let us assume regularity of $\phi$ at the horizon $(U=0)$ or at the origin $(R=0)$. Thus, we must have $\phi^{\prime}=0$ there. Without loss of generality, one can make $\phi>0$ there, but not $\phi=0$ (in this case, $\phi$ is zero everywhere). Then the quantity $\left(U R \phi^{\prime}\right)^{\prime}$ is positive as we move outside the horizon or the origin. One can write

$$
\left.U R \phi^{\prime}\right|_{x_{0}} ^{x}=\int_{x_{0}}^{x} d x\left(M^{2} U R \phi\right)
$$

where the lower limit $x_{0}$ represents the horizon or the origin. As $\phi^{\prime}$ is positive (according to the previous equation), we obtain

$$
\left.U R \phi^{\prime}\right|_{x_{0}} ^{x}>\phi\left(x_{0}\right) \int_{x_{0}}^{x} d x\left(M^{2} U R\right) .
$$

\footnotetext{
${ }^{5}$ The author is grateful to Professor Don Page for explaining this point.
} 
If the spacetime is asymptotically flat, then $U \rightarrow 1$ and $R \rightarrow x$ as $x \rightarrow \infty$. So we can consider the dominant contribution to (26) for large $x$, as follows:

$$
x \phi^{\prime}>\frac{1}{2} M^{2} x^{2} \phi\left(x_{0}\right) .
$$

Consequently, we have $\phi^{\prime}>(1 / 2) M^{2} x \phi\left(x_{0}\right)$, which means that the scalar field grows too fast, so it will prevent the spacetime from being asymptotically flat by the back reaction. Thus, there is no static circularly symmetric asymptotically flat solution for the non-trivial massive scalar field minimally coupled with Einstein gravity (if the scalar field is regular everywhere). Also, the same result is valid for the asymptotically De Sitter and anti-De Sitter cases. The asymptotically anti-De Sitter spacetime is particularly interesting because it can be regarded as some asymptotic solution (e.g., static BTZ solution) for a black hole solution with massive scalar field and $\Lambda<0$.

This result implies that both the ansatz (19) and (21) are incompatible with the solution assumed at the infinity, which is asymptotically flat, because they are approximate solutions with regular scalar field at the origin and at the horizon, respectively. Thus the integration carried out from infinity should correspond to a solution with non-regular scalar field at the origin. In this case, there is a naked singularity.

\section{Conclusions}

We have investigated circularly symmetric static solutions of gravity minimally coupled to massive scalar field in three dimensions, and then we presented a numerical solution containing a naked singularity and consistent with the no-hair theorems and no-black hole theorem (see [33]) in three dimensions.

By the results of subsection $§ 3.1$, the unique possibility to find a non-trivial black hole solution is to consider the cases with non-regular scalar field at the origin. Among these cases, there are two classes of solutions:

(i) Asymptotically flat solutions;

(ii) Solutions which are not asymptotically flat.

The first group are covered by the numerical integration procedure described in the preceding section. The second group (ii) was not considered in this paper. Notice that in principle one can not guess suitable initial conditions in any region.

\section{Acknowledgments.}

I would like to thank Professor V.P. Frolov for invaluable discussions and to University of Alberta for warm hospitality and support. I also acknowledge Professor J.A. Helayël-Neto and Professor D. Page for useful discussions. This work was done with support from CNPq, a Brazilian Government institution that promotes development in science and technology. 


\section{Appendix}

\section{Gravity with dynamical torsion in three dimensions}

Let us consider Dirac fermions in the Riemann-Cartan three-dimensional spacetime, which can be described by the action $(\hbar=c=1)$

$$
S_{\psi}=\int d^{3} x \sqrt{-g}\left\{\frac{1}{\kappa} \tilde{R}+\frac{i}{2}\left(\bar{\psi} \gamma^{\mu} \tilde{\nabla}_{\mu} \psi-\tilde{\nabla}_{\mu} \bar{\psi} \gamma^{\mu} \psi\right)-m \bar{\psi} \psi\right\}
$$

where the objects with tilde are constructed with non-trivial torsion ( $R$ is the Ricci scalar and $\nabla_{\mu}$ is the Riemannian covariant derivative),

$$
T_{\alpha \beta}^{\mu}:=\tilde{\Gamma}_{\alpha \beta}^{\mu}-\tilde{\Gamma}_{\beta \alpha}^{\mu} .
$$

We adopt $R_{\nu \alpha \beta}^{\mu}:=\partial_{\alpha} \Gamma^{\mu}{ }_{\nu \beta}-\ldots$. The covariant gamma matrices are defined in the usual way, $\gamma^{\mu}:=e^{\mu}{ }_{a} \gamma^{a}\left(e^{\mu}{ }_{a}\right.$ are the dreibeins). By the procedure $\nabla_{\mu} \rightarrow \tilde{\nabla}_{\mu}$, we consider minimal coupling between torsion and fermions. Straightforward calculations enable us to write ${ }^{6}$

$$
S_{\psi}=\int d^{3} x \sqrt{-g}\left\{\frac{1}{\kappa} \tilde{R}+\frac{i}{2}\left(\bar{\psi} \gamma^{\mu} \nabla_{\mu} \psi-\nabla_{\mu} \bar{\psi} \gamma^{\mu} \psi\right)-m \bar{\psi} \psi+\frac{i}{8} T_{\mu \nu \alpha} \bar{\psi} \gamma^{[\mu} \gamma^{\nu} \gamma^{\alpha]} \psi\right\}
$$

Here the brackets mean total antisymmetrization. We can choose the following gamma matrices basis, $\gamma^{a}$, in terms of the Pauli matrices, $\sigma^{i}$,

$$
\gamma^{0}:=-i \sigma^{3} ; \quad \gamma^{1}:=\sigma^{1} ; \quad \gamma^{2}:=\sigma^{2}
$$

They satisfy

$$
\left\{\gamma^{\mu}, \gamma^{\nu}\right\}=2 g^{\mu \nu} \hat{1}
$$

where $g^{\mu \nu}$ has signature +1 . After some algebra, one can achieve

$$
\gamma^{[\mu} \gamma^{\nu} \gamma^{\alpha]}=\frac{1}{\sqrt{-g}} \gamma^{0} \gamma^{1} \gamma^{2} \varepsilon^{\mu \nu \alpha}=\frac{1}{\sqrt{-g}} \varepsilon^{\mu \nu \alpha}
$$

where $\varepsilon^{\mu \nu \alpha}$ is the antisymmetrization symbol $\left(\varepsilon^{012}:=+1\right)$. Thus, taking into account that the totally antisymmetric part of the torsion in three dimensions is related to the pseudoscalar $\chi:=\varepsilon^{\mu \nu \alpha} T_{\mu \nu \alpha}$ (which has weight -1 ), we can write the interacting action as

$$
S_{I}=\int d^{3} x \sqrt{-g} \frac{i}{8} T_{\mu \nu \alpha} \bar{\psi} \gamma^{[\mu} \gamma^{\nu} \gamma^{\alpha]} \psi=\int d^{3} x \sqrt{-g} \frac{i}{8} \bar{\psi} \frac{1}{\sqrt{-g}} \chi \psi
$$

where $\frac{1}{\sqrt{-g}} \chi$ is a scalar. Thus, in three dimensions, the minimal coupling between fermions and torsion gives a Yukawa coupling, and the torsion component which couples with fermions is described by the pseudo-scalar $\chi(x)$ [35].

Now we consider only the $\chi$-component of torsion in the description of gravity with dynamical torsion. We choose the action

$$
S=\int d^{3} x \sqrt{-g}\left\{\frac{1}{\kappa} R-\nabla_{\mu} \chi \nabla^{\mu} \tilde{\chi}-M^{2} \chi \tilde{\chi}\right\}
$$

\footnotetext{
${ }^{6}$ See [34] for detailed calculations, and [35] for the Minkowski background case in three dimensions.
} 
where $\tilde{\chi}$ is the dual of $\chi, \tilde{\chi}:=-\frac{1}{g} \chi$, such that the action is a scalar. After the variable change

$$
\chi(x) \rightarrow \phi(x)=\frac{1}{\sqrt{-g}} \chi(x),
$$

the action can be written as a scalar-metric action (2). Notice that the conventional theories with dynamical torsion are usually described by an action in a different way. For instance, one considers the Lagrangian quadratic in torsion and total curvature, which are the field strength of tetrads and spin connection, respectively (see [5]). After some manipulation, it is possible to write the usual Lagrangian in terms of the metric and the torsion, such that the Lagrangian is written as a torsion component plus a purely Riemannian one. In the theory (2) the dynamical term for the torsion was introduced by hand, and not in the natural way from to the total curvature squared. For theories of dynamical torsion in this scenario, see, for example, the papers in the Refs. [36, 6].

\section{References}

[1] R. Wald, General Relativity, Chicago University Press, Chicago, USA (1984).

[2] V.P. Frolov and I.D. Novikov, Black Hole Physics: Basic concepts and new developments, Kluwer Academic, Dordrecht, Netherlands (1998).

[3] S.W. Hawking, Particle Creation by Black Holes, Commun. Math. Phys. 43 (1975) 199-220.

[4] J.M. Bardeen, B. Carter and S.W. Hawking, The Four Laws of Black Hole Mechanics, Commun. Math. Phys. 31 (1973) 161-170.

[5] F.W. Hehl, P. von der Heyde, G.D. Kerlick and J.M. Nester, General relativity with spin and torsion: foundations and prospects, Rev. Mod. Phys. 48 (1976) 393; F.W.Hehl, Y.Ne'eman, J.Nitsch and P. von der Heyde, Short Range Confining Component in a Quadratic Poincaré Gauge Theory of Gravitation Phys. Lett. B 78 (1978) 102-106.

[6] I.L. Shapiro, Physical Aspects of the Space-time Torsion, Phys. Rep. 357 (2001) 113. [hep-th/1013093]

[7] Peter Baekler, The unique spherically symmetric solution of the $U_{4}$-Theory of Gravity in the teleparallelism limit, Phys. Lett. B 94 (1980) 44-50.

[8] Peter Baekler, A spherically symmetric vacuum solution of the quadratic Poincaré Gauge Field Theory of Gravitation with Newtonian and confinement potentials, Phys. Lett. B 99 (1981) 329-332.

[9] P. Baekler and F.W. Hehl, A charged Taub-NUT metric with torsion: A new axially symmetric solution of the Poincaré gauge field theory, Phys. Lett. A 100 (1984) 392396.

[10] M. Bañados, C. Teitelboim and J. Zanelli, The Black Hole in Three Dimensional Spacetime, Phys. Rev. Lett. 69 (1992) 1849. 
[11] S. Carlip. The (2+1)-dimensional black hole, Class. Quantum Grav. 12 (1995) 28532879.

[12] G.T. Horowitz and D.L. Welch, String Theory Formulation of the Three-Dimensional Black Hole, Phys. Rev. Lett. 71 n.3 (1993) 328-331.

[13] R.B. Mann and S.F. Ross, Gravitationally Collapsing Dust in (2+1)-Dimensions, Phys. Rev. D 47 (1993) 3319-3322.

[14] N. Cruz and J. Zanelli, Stellar Equilibrium in (2+1) Dimensions, Class. Quantum Grav. 12 (1995) 975-982.

[15] G. Oliveira-Neto, Self-similar collapse of a massless scalar field in three dimensions, gr-qc/0105077.

[16] C. Martínez, C. Teitelboim and J. Zanelli, Charged rotating black hole in three spacetime dimensions, Phys. Rev. D 61 (2000) 104013.

[17] G. Clément, Classical solutions in three-dimensional Einstein-Maxwell cosmological gravity, Class. Quant. Grav. 10 (1993) L49.

[18] E.W. Hirschmann and D.L. Welch, Magnetic solutions to $2+1$ gravity, Phys. Rev. D $53(1996) 5579$.

[19] M. Cataldo and P. Salgado, Static Einstein-Maxwell solutions in (2+1)-dimensions, Phys. Rev. D 54 (1996) 2971.

[20] Óscar J.C. Dias and José P.S. Lemos, Rotating magnetic solution in three dimensional Einstein gravity, JHEP 0201 (2002) 006.

[21] Óscar J.C. Dias and José P.S. Lemos, Static and rotating electrically charged black holes in three-dimensional Brans-Dicke gravity theories, Phys. Rev. D 64 (2001) 064001.

[22] G. Clément and A. Fabbri, The gravitating si model in 2+1 dimensions: black hole solutions, Class. Quantum Grav. 16 (1999) 323-341; G. Clément and A. Fabbri, The cosmological gravitating $\sigma$ model: solitons and black holes, Class. Quantum Grav. 17 (2000) 2537-2545.

[23] K.C.K. Chan and R.B. Mann, Static charged black holes in (2+1)-dimensional dilaton gravity, Phys. Rev. D 50 n.10 (1994) 6385-6393.

[24] K.C.K. Chan and R.B. Mann, Spinning black holes in (2+1)-dimensional string and dilaton gravity, Phys. Lett. B 371 (1996) 199-205.

[25] J. Gegenberg, C. Martínez and R. Troncoso, A finite action for three dimensional gravity with a minimally coupled scalar field, hep-th/0301190.

[26] A.A. Garcia, F.W. Hehl, C. Heinicke and A. Macias, Exact Vacuum solution of a $(2+1)$ dimensional Poincare gauge theory: BTZ solution with torsion, gr-qc/0302097.

[27] A.A. Sousa and J.W. Maluf, Black holes in 2+1 teleparallel theories of gravity, grqc/0301079. 
[28] D. Sudarsky, A simple proof of a no-hair theorem in Einstein-Higgs theory, Class. Quant. Grav. 12 (1995) 579-584.

[29] S. Giddings, J. Abbott and K. Kuchar, Einstein's Theory in a Three-dimensional SpaceTime, Gen. Rel. Grav. 16 n.8 (1984) 751-775; S. Deser, R. Jackiw and G. t'Hooft, Three-Dimensional Einstein Gravity: Dynamics of Flat Space, Annals Phys.152 (1984) 220.

[30] J.D. Bekenstein, Transcendence of the Law of Baryon-Number Conservation in Black Hole Physics, Phys. Rev. Lett. 28 n.7 (1972) 452-455.

[31] Y.N. Obukhov and E.J. Vlachynsky, Einstein-Proca model: spherically symmetric solutions, Ann. Phys. 8 (1999) 497-510; M. Toussaint, A Numeric Solution for Einstein's Gravitational Theory with Proca Matter and Metric-Affine Gravity, Gen. Rel. Grav. 32 n.9 (2000) 1689-1709.

[32] A. Heck, Introduction to Maple, (Springer-Verlag, 2003).

[33] Daisuke Ida, No Black Hole Theorem in Three Dimensional Gravity, Phys. Rev. Lett. 85 n.18 (2000) 3758-3760.

[34] V. de Sabbata and M. Gasperini, Introduction to Gravitation, World Scientific, Singapore (1985).

[35] J.L. Boldo, J.A. Helayël-Neto and N. Panza, Propagating Torsion in 3D-Gravity and Dynamical Mass Generation, Class. Quantum Grav. 19 (2002) 2201.

[36] G. de Berredo-Peixoto, J.A. Helayël-Neto and I.L. Shapiro, On the Consistency of a Fermion-Torsion Effective Theory, JHEP 02 (2000) 003.

$* * *$

Figure caption: Numerical integration from infinity, assuming the asymptotically flat ansatz. The curves $G, F$ and $U$ correspond to the functions $g(r), f(r)$ and $\phi(r)$, respectively. 


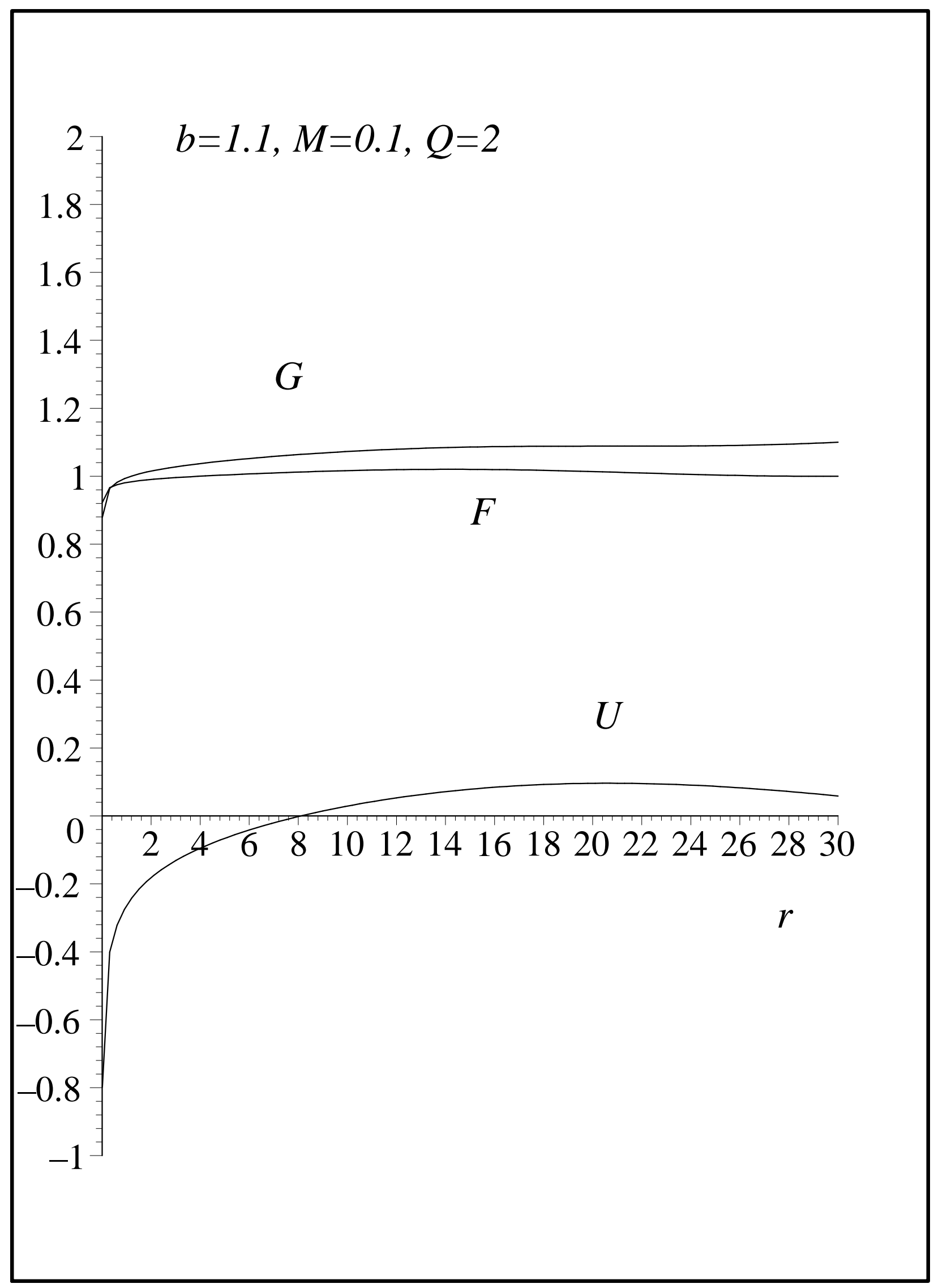

